\title{
HOMOCLINIC CHAOS IN GENERALIZED HENON-HEILES SYSTEM
}

\author{
S. KASPERCZUK \\ Institute of Physics, Pedagogical University \\ Pl. Słowiański 6, 65-069 Zielona Góra, Poland
}

(Received June 27, 1995)

This paper considers the generalized Henon-IIeiles system, defined by the Hamiltonian $H=\left(p_{1}^{2}+p_{2}^{2}+A q_{1}^{2}+B q_{2}^{2}\right) / 2+C q_{1}^{2} q_{2}+D q_{2}^{3}$. Melnikov's method is used to prove the existence of nondegenerate homoclinic orbits near two integrable cases: (o) $C=0 ; A, B, D$ arbitrary; (i) $A=B$; $C=3 D$. The existence of such orbits precludes the existence of analytic second integrals.

PACS numbers: 05.45.+b

\section{Introduction}

The generalized Henon-Ieiles IIamiltonian

$$
H=\frac{1}{2}\left(p_{1}^{2}+p_{2}^{2}+A q_{1}^{2}+B q_{2}^{2}\right)+C q_{1}^{2} q_{2}+D q_{2}^{3}
$$

is well known from applications in stellar dynamics, statistical mechanics and quantum mechanics (cf. [1-3]). In case $A=B=C=-3 D=1$ the IIamiltonian (1) reduces to its standard form [4]. This system is known to be integrable when

(o) $C=0 ; A, B, D$ arbitrary;

(i) $A=B ; C=3 D$;

(ii) $A, B$ arbitrary; $D=2 C$;

(iii) $\mathrm{B}=16 \mathrm{~A} ; 3 \mathrm{D}=16 \mathrm{C}$.

The second integrals for the sets (i)-(iii) have long been known (cf. $[5,6]$ ).

Since the unperturbed (integrable) and perturbed IIamiltonian systems are close, one might expect that the solutions will also be close, but this is not generally the case. In fact, arbitrarily small perturbations of a system can cause radical qualitative changes in the topological structure of the phase space. The most important type of orbits in IIamiltonian systems are homoclinic orbits. The concept and the terminology of homoclinic and heteroclinic solutions goes back to Poincaré [7]. The homoclinic solution is forward and backward asymptotic to the periodic orbit. In Hamiltonian systems the existence of nondegencrate homoclinic orbits connecting the hyperbolic periodic orbit leads to the Smale horseshoe (see e.g. [8]). The complex behaviour of nearby orbits implies the nonexistence of second analytical 
integrals for the flow. Hence the presence of a nondegenerate homoclinic orbit in a Hamiltonian system is enough to guarantee the existence of chaotic behaviour. We have no general method for proving the existence of a nondegenerate homoclinic orbit in a given Hamiltonian system. The Poincaré-Melnikov method (cf. [9-11]) can often be used to show that certain degenerate homoclinic orbits become nondegenerate under perturbations. However, to apply this method one must already have the existence of a homoclinic orbit in the unperturbed system. Unfortunately, proving transversal intersections of stable and unstable Lagrangian manifolds of the hyperbolic orbit is nontrivial in most problems.

In this paper we consider the generalized Ilenon-IIeiles system (1) near two integrable cases $(\mathrm{o})=(A, B, 0, D)$ and $(\mathrm{i})=(A, A, C, C / 3)$. Our aim is to prove that for infinitesimal deformations of the sets (o) and (i):

$$
\begin{aligned}
& \mathrm{o}_{1}:(A, B, 0, D) \rightarrow(A, B, \mu, D) ; \mathrm{i}_{1}:(A, A, C, C / 3) \rightarrow(A+\mu, A, C, C / 3) ; \\
& \mathrm{i}_{2}:(A, A, C, C / 3) \rightarrow(A, A+\mu, C, C / 3) ; \mathrm{i}_{3}:(A, A, C, C / 3) \rightarrow \\
& (A, A, C+\mu, C / 3) ; \mathrm{i}_{4}:(A, A, C, C / 3) \rightarrow(A, A, C, C / 3+\mu) ; \\
& \mathrm{i}_{5}:(A, A, C, C / 3) \rightarrow(A+\mu, A, C+\mu, C / 3) ; \mathrm{i}_{6}:(A, A, C, C / 3) \rightarrow \\
& (A, A+\mu, C+\mu, C / 3) ; \mathrm{i}_{7}:(A, A, C, C / 3) \rightarrow(A+\mu, A, C, C / 3+\mu) ; \\
& \mathrm{i}_{8}:(A, A, C, C / 3) \rightarrow(A, A+\mu, C, C / 3+\mu) ; \mathrm{i}_{9}:(A, A, C, C / 3) \rightarrow \\
& (A+\mu, A, C+\mu, C / 3+\mu) ; \mathrm{i}_{10}:(A, A, C, C / 3) \rightarrow \\
& (A, A+\mu, C+\mu, C / 3+\mu) ; \mathrm{i}_{11}:(A, A, C, C / 3) \rightarrow(A+\mu, A+\mu, C+\mu, C / 3) ; \\
& \mathrm{i}_{12}:(A, A, C, C / 3) \rightarrow(A+\mu, A+\mu, C, C / 3+\mu) ; \\
& \mathrm{i}_{13}:(A, A, C, C / 3) \rightarrow(A+\mu, A+\mu, C+\mu, C / 3+\mu)
\end{aligned}
$$

the Henon-Heiles system has transversal homoclinic orbits. We extend the result of a paper of Holmes [12] in which he has proved the existence of transversal homoclinic orbits for case $i_{8}$.

\section{Homoclinic chaos}

We begin with considering two-degree of freedom Hamiltonian systems with perturbations that break an $S^{1}$ phase symmetry. These systems have the following form:

$$
\begin{aligned}
& \mathrm{d} x / \mathrm{d} t=J D_{\boldsymbol{x}} H^{0}(\boldsymbol{x}, I)+\mu J D_{\boldsymbol{x}} H^{1}(\boldsymbol{x}, I, u), \\
& \mathrm{d} I / \mathrm{d} t=-\mu D_{u} H^{1}(\boldsymbol{x}, I, u), \\
& \mathrm{d} u / \mathrm{d} t=D_{I} H^{0}(\boldsymbol{x}, I)+\mu D_{I} H^{1}(\boldsymbol{x}, I, u),
\end{aligned}
$$

where $x=\left(x_{1}, x_{2}\right), I$ is an action and $u$ is its conjugate angle, $J=\left\|\begin{array}{rr}0 & -1 \\ 1 & 0\end{array}\right\|$, $\mu$ is a small parameter, $D_{x}=\left(D_{x_{1}}, D_{x_{2}}\right)$ represents partial derivative with respect 
to $x, D_{I}$ represents the partial derivative with respect to $I$, and $D_{u}$ represents the partial derivative with respect to $u$. The system (4) is derived from the Hamiltonian

$$
H(\boldsymbol{x}, I, u)=H^{0}(\boldsymbol{x}, I)+\mu H^{1}(\boldsymbol{x}, I, u) .
$$

The unperturbed system

$$
\begin{aligned}
\mathrm{d} x / \mathrm{d} t & =J D_{\boldsymbol{x}} H^{0}(\boldsymbol{x}, I), \\
\mathrm{d} I / \mathrm{d} t & =0, \\
\mathrm{~d} u / \mathrm{d} t & =D_{I} H^{0}(x, I),
\end{aligned}
$$

is completely integrable. We assume that Eq. (6a) possesses a homoclinic orbit $\boldsymbol{x}^{*}$, asymptotic to the hyperbolic point $x^{0}$. In full $(x, I, u)$ phase space each equilibrium point $\boldsymbol{x}^{0}$ corresponds to a periodic orbit $C_{\boldsymbol{x}^{0}}$ parametrized by the solution

$$
x=x^{0}, I=I^{0}, u=D_{I} H^{0}\left(x^{0}, I^{0}\right)+u^{0} .
$$

Each hyperbolic orbit $C_{\not^{\circ}}$ inside the corresponding level surface of the Hamiltonian $H^{0}$ possesses two-dimensional stable and unstable Lagrangian manifolds $W^{s}\left(x^{0}\right)$ and $W^{u}\left(x^{0}\right)$. The parts of the stable and unstable manifolds which do not extend to infinity coincide to form a two-dimensional manifold $W\left(x^{0}\right)$. As shown in [11], the invariant hyperbolic orbit $C_{x^{0}}$ persists under perturbations with stable and unstable manifolds. Any trajectory on the perturbed stable manifold $W^{s}\left(\boldsymbol{x}^{\mu}\right)$ stays $\mathrm{O}(\mu)$ close to a trajectory on the unperturbed stable manifold $W^{s}\left(x^{0}\right)$ and any trajectory on the perturbed unstable manifold $W^{u}\left(x^{\mu}\right)$ stays $\mathrm{O}(\mu)$ close to a trajectory on the unperturbed unstable manifold $W^{u}\left(x^{0}\right)$, both on the time interval $\left(-t^{0}, t^{0}\right)$ for any positive $t^{0}$. Since the unperturbed manifolds $W^{s}\left(x^{0}\right)$ and $W^{u}\left(x^{0}\right)$ are not transverse, we thus expect the perturbed manifolds $W^{s}\left(x^{\mu}\right)$ and $W^{u}\left(\boldsymbol{x}^{\mu}\right)$ to intersect transversely creating the homoclinic tangle.

We check for the existence of transverse homoclinic intersections using the Poincaré-Melnikov method. This standard method was first developed by Holmes and Marsden [9] and Robinson [10]. The Poincaré-Melnikov function measures the separation between the perturbed manifolds, the manifolds $W^{s}\left(\boldsymbol{x}^{\mu}\right)$ and $W^{u}\left(\boldsymbol{x}^{\mu}\right)$ intersect where this distance vanishes. We use the unperturbed manifold $W\left(\boldsymbol{x}^{0}\right)$ as the framework for parametrizing the manifolds $W^{s}\left(x^{\mu}\right)$ and $W^{u}\left(x^{\mu}\right)$ as follows. At every point $y=(\boldsymbol{x}, I, u)$ on the homoclinic manifold $W\left(x^{0}\right)$ we consider the normal $\boldsymbol{N}(y)$ to $W\left(x^{0}\right)$. Let the point $y^{\mu, s}$ (resp. $y^{\mu, u}$ ) be unique intersection point of the normal $N(y)$ and the stable $W^{s}\left(x^{\mu}\right)$ (resp. unstable $W^{u}\left(x^{\mu}\right)$ ) manifold inside $\mathrm{O}(\mu)$ neighbourhood of $y$. We define the separation distance of the manifolds $W^{s}\left(x^{\mu}\right)$ and $W^{u}\left(x^{\mu}\right)$ at the point $y$ as

$$
\mathrm{d}(y, \mu)=\left(y^{\mu, s}-y^{\mu, u} \mid N(y)\right) /|N(y)|,
$$

where $(\mid)$ denotes the Euclidean scalar product and || denotes the corresponding Euclidean norm. The distance $d(y, \mu)$ can be expanded in Taylor series (cf. $[9,10])$ to become

$$
d(y, \mu)=\mu M\left(u^{0}\right) /|\boldsymbol{X}(y)|+\mathrm{O}\left(\mu^{2}\right) .
$$

The expression

$$
M\left(u^{0}\right)=\left.\int_{-\infty}^{\infty}\left(d H^{0} \mid \boldsymbol{X}^{1}\right)\right|_{x^{*}} \mathrm{~d} t
$$


is called the Poincaré-Melnikov function. Ilere $\boldsymbol{X}^{0}=\left(J D_{\boldsymbol{x}} H^{0}, D_{I} H^{0}, 0\right)$, $\boldsymbol{X}^{1}=\left(J D_{\boldsymbol{x}} H^{1},-D_{u} H^{1}, D_{I} I^{1}\right), d H^{0}=\left(D_{\boldsymbol{x}} H^{0}, D_{I} H^{0}, 0\right)$, and $\left.\left(d H^{0} \mid \boldsymbol{X}\right)\right|_{x^{*}}$ is evaluated at the homoclinic orbit $\boldsymbol{x}^{*}$. Since $|\boldsymbol{X}(y)|=\mathrm{O}(1), M\left(u^{0}\right)$ provides a good measure of the separation of the manifolds $W^{s}\left(x^{\mu}\right)$ and $W^{u}\left(x^{\mu}\right)$. To show that transverse homoclinic orbits occur for $\mu \neq 0$ we only need to show that the Poincaré-Melnikov function $M\left(u^{0}\right)$ has simple zeros. Via the Smale-Birkhoff homoclinic theorem [8] this situation leads to chaotic dynamics in the vicinity of the homoclinic orbits.

\subsection{Case (o)}

We assume that the Hamiltonian (1) depends on a small parameter $\mu=C$ :

$$
H=K_{1}\left(p_{1}, q_{1}\right)+K_{2}\left(p_{2}, q_{2}\right)+\mu I^{1}\left(q_{1}, q_{2}\right),
$$

where $K_{1}=\left(p_{1}^{2}+A q_{1}^{2}\right) / 2, K_{2}=\left(p_{2}^{2}+B q_{2}^{2}+2 D q_{2}^{3}\right) / 2$ and $H^{1}=q_{1}^{2} q_{2}$. The equations of motion for $H^{0}=K_{1}+K_{2}$ are

$$
\begin{aligned}
& \mathrm{d} p_{1} / \mathrm{d} t=-A q_{1}, \quad \mathrm{~d} q_{1} / \mathrm{d} t=p_{1}, \\
& \mathrm{~d} p_{2} / \mathrm{d} t=-B q_{2}-3 D q_{2}^{2}, \quad \mathrm{~d} q_{2} / \mathrm{d} t=p_{2} .
\end{aligned}
$$

The system $(12 \mathrm{~b})$ has two equilibrium points $(0,0)$ and $(0,-B / 3 D)$. The equilibrium point $\left(p_{2}^{0}, q_{2}^{0}\right)=(0,-B / 3 D)$ is a hyperbolic saddle point, there is a homoclinic orbit $\left(p_{2}^{*}, q_{2}^{*}\right)$ joining the saddle point to itself. An elementary integration implies

$$
\begin{aligned}
& p_{2}^{*}=B^{3 / 2}(2 D)^{-1} \tanh \left(-B^{1 / 2} t / 2\right) \operatorname{sech}^{2}\left(-B^{1 / 2} t / 2\right), \\
& q_{2}^{*}=B(6 D)^{-1}\left[1-3 \tanh ^{2}\left(-B^{1 / 2} t / 2\right)\right] .
\end{aligned}
$$

Employing the canonical transformation $\left(p_{1}, q_{1}\right) \rightarrow(I, u)$ :

$$
p_{1}=\left(4 A I^{2}\right)^{1 / 4} \cos u, \quad q_{1}=\left(4 I^{2} / A\right)^{1 / 4} \sin u
$$

$K_{1}$ becomes

$$
K_{1}=A^{1 / 2} I \text {. }
$$

The system (12a) contains periodic orbits $(I, u)=\left(I^{0}, u^{0}+A^{1 / 2} t\right)$. Thus the Hamiltonian $H^{0}$ for $K_{2}=B^{3} / 54 D^{2}$ and $I^{0} \neq 0$ has the hyperbolic closed orbit with the homoclinic connection. In terms of variables $\left(I, p_{2}, u, q_{2}\right) \mathrm{Eq}$. (10) gives

$$
\begin{gathered}
M_{0}\left(u^{0}\right)=E \cos \left(u^{0}\right) \int_{-\infty}^{\infty} \tanh (b t) \operatorname{sech}^{2}(b t) \sin \left(A^{1 / 2} t\right) \mathrm{d} t \\
-E \sin \left(u^{0}\right) \int_{-\infty}^{\infty} \tanh (b t) \operatorname{sech}^{2}(b t) \cos \left(A^{1 / 2} t\right) \mathrm{d} t,
\end{gathered}
$$

where $E=\left(h-h_{1}\right) B^{1 / 2}(A D)^{-1}, b^{2}=B / 4, h$ is a total energy, $h_{1}=B^{3} / 54 A^{2}$. The second integral in (16) is easily calculated, the first must be evaluated by the method of residues (cf. [13]). We obtain by standard methods

$$
M_{0}\left(u^{0}\right)=4 \pi(A D)^{-1}\left(h-B^{3} / 54 D^{2}\right)^{1 / 2} \operatorname{cosech}\left[\pi(A / B)^{1 / 2}\right] \cos \left(u^{0}\right) .
$$

From Eq. (17) one can see that the Poincaré-Melnikov function $M_{0}\left(u^{0}\right)$ has simple zeros. This proves the existence of the homoclinic chaos for sufficiently small $C \neq 0$ and $h>h_{1}$. 


\subsection{Case (i)}

For the integrable case (i) the Hamiltonian (1) separates with

$$
\begin{aligned}
& x=2^{-1 / 2}\left(q_{1}+q_{2}\right), \quad y=2^{-1 / 2}\left(q_{1}-q_{2}\right), \\
& p_{x}=2^{-1 / 2}\left(p_{1}+p_{2}\right), \quad p_{y}=2^{-1 / 2}\left(p_{1}-p_{2}\right),
\end{aligned}
$$

into

$$
H=L_{1}\left(p_{x}, x\right)+L_{2}\left(p_{y}, y\right)
$$

where $L_{1}=\left(p_{x}+A x^{2}\right) / 2+2^{1 / 2} C x^{3} / 3, L_{2}=\left(p_{y}+A y^{2}\right) / 2-2^{1 / 2} C y^{3} / 3$. The system (19) describes two uncoupled oscillators, each having a homoclinic loop, joining a hyperbolic saddle point. The energy on each homoclinic loop is $h^{*}=$ $A^{3} / 12 C^{2}$. Ilence the homoclinic orbit $\left(p_{x}^{*}, x^{*}\right)$ is given by

$$
p_{x}^{* 2}=A^{3}\left(6 C^{2}\right)^{-1}-2^{3 / 2} C x^{* 3}-A x^{* 2} .
$$

An elementary integration implies

$$
\begin{aligned}
& p_{x}^{*}(t)=3 A\left(2^{3 / 2} C\right)^{-1} \tanh \left[-\left(2^{1 / 2} C / 6\right)^{1 / 2} t\right] \operatorname{sech}^{2}\left[-\left(2^{1 / 2} C / 6\right)^{1 / 2} t\right], \\
& x^{*}(t)=A\left(2^{3 / 2} C\right)^{-1}-3 A\left(2^{3 / 2} C\right)^{-1} \tanh ^{2}\left[-\left(2^{1 / 2} C / 6\right)^{1 / 2} t\right] .
\end{aligned}
$$

For $L_{2}=h_{2}, 0<h_{2}<h^{*}$, the system (19) has periodic solutions. Let $\left(a_{1}, a_{2}, a_{3}\right)$ be a set of roots of the equation $y^{3}-3 A\left(8^{1 / 2} C\right)^{-1} y^{2}+3 h_{2}\left(2^{1 / 2} C\right)^{-1}=0$. Then it follows that

$$
y\left(t, h_{2}\right)=a_{i}+\left(a_{j}-a_{i}\right) \operatorname{sn}^{2}(t, k),
$$

where $k^{2}=\left(a_{i}-a_{j}\right) /\left(a_{i}-a_{s}\right), a_{i} \neq a_{s}, \operatorname{sn}(t, k)$ is Jacobi's elliptic function, $k$ is modulus of $\operatorname{sn}(l, k)$. For simplicity we shall assume that $h_{2} / h^{*}$ is sufficiently small and (22) is well approximated by $y=\left(h_{2} / A\right)^{1 / 2} \cos \left(A^{1 / 2} t\right)$. Employing the canonical transformation $p_{y}=\left(4 A I^{2}\right)^{1 / 4} \cos u, y=\left(4 I^{2} / A\right)^{1 / 4} \sin u, L_{2}$ becomes $L_{2}=A^{1 / 2} I$. Thus for sufficiently small $L_{2}$ and $L_{1}=h^{*}$ the system (20) have the degenerate homoclinic orbits $\left(p_{x}^{*}, x^{*}, I, u\right)$, where $\left(p_{x}^{*}, x^{*}\right)$ is given by $(21)$ and $(I, u)=\left(I^{0}, u^{0}+A^{1 / 2} t\right)$.

Now we consider the following deformations of the set $(\mathrm{i})=(A, A, C, C / 3)$ :

$$
\mathrm{i}_{1}:(A, A, C, C / 3) \rightarrow(A+\mu, A, C, C / 3) ; \mathrm{i}_{2}:(A, A, C, C / 3) \rightarrow
$$$$
(A, A+\mu, C, C / 3) ; \mathrm{i}_{3}:(A, A, C, C / 3) \rightarrow(A, A, C+\mu, C / 3) ;
$$$$
\mathrm{i}_{4}:(A, A, C, C / 3) \rightarrow(A, A, C, C / 3+\mu) ; \mathrm{i}_{5}:(A, A, C, C / 3) \rightarrow
$$$$
(A+\mu, A, C+\mu, C / 3) ; \mathrm{i}_{6}:(A, A, C, C / 3) \rightarrow(A, A+\mu, C+\mu, C / 3) ;
$$

$$
\begin{aligned}
& \mathrm{i}_{7}:(A, A, C, C / 3) \rightarrow(A+\mu, A, C, C / 3+\mu) ; \mathrm{i}_{8}:(A, A, C, C / 3) \rightarrow \\
& (A, A+\mu, C, C / 3+\mu) ; \mathrm{i}_{9}:(A, A, C, C / 3) \rightarrow(A+\mu, A, C+\mu, C / 3+\mu) ;
\end{aligned}
$$$$
\mathrm{i}_{10}:(A, A, C, C / 3) \rightarrow(A, A+\mu, C+\mu, C / 3+\mu) ; \mathrm{i}_{11}:(A, A, C, C / 3) \rightarrow
$$$$
(A+\mu, A+\mu, C+\mu, C / 3) ; \mathrm{i}_{12}:(A, A, C, C / 3) \rightarrow(A+\mu, A+\mu, C, C / 3+\mu) ;
$$$$
\mathrm{i}_{13}:(A, A, C, C / 3) \rightarrow(A+\mu, A+\mu, C+\mu, C / 3+\mu) .
$$ 
We show that for the deformations (23) the generalized Henon-Heiles system has transverse homoclinic orbits. Let $M_{k}, k=1,2, \ldots, 13$, denote the PoincaréMelnikov functions for the deformations $\mathrm{i}_{k}, k=1,2, \ldots, 13$. Then one can easily show that

$$
\begin{aligned}
& M_{2}=-M_{1}, M_{4}=-3 M_{3}, M_{5}=M_{1}+M_{3}, M_{6}=-M_{1}+M_{3}, \\
& M_{7}=M_{1}-3 M_{3}, M_{8}=-M_{1}-M_{3}, M_{9}=M_{1}-2 M_{3}, M_{10}=-M_{1}-2 M_{3}, \\
& M_{11}=M_{3}, M_{12}=-3 M_{1}, M_{13}=-2 M_{3},
\end{aligned}
$$

where

$$
\begin{aligned}
& M_{1}\left(u^{0}\right)=\int_{-\infty}^{\infty} p^{*}(t) \sin \left(A^{1 / 2} t-u^{0}\right) \mathrm{d} t \\
& M_{3}\left(u^{0}\right)=-2^{-1 / 2} \int_{-\infty}^{\infty} p^{*}(t) x^{*}(t) \sin \left(A^{1 / 2} t-u^{0}\right) \mathrm{d} t .
\end{aligned}
$$

Inserting (21) into (25) and (26), we obtain

$$
\begin{aligned}
& M_{1}\left(u^{0}\right)=-(3 \pi / 32)(A / C)^{2}\left[2 A\left(h-h^{*}\right)\right]^{1 / 2} \operatorname{sech}(g) \cos \left(u^{0}\right), \\
& M_{3}\left(u^{0}\right)=(3 \pi / 32)(A / C)^{2}\left[2 A\left(h-h^{*}\right)\right]^{1 / 2}(1-g) \operatorname{sech}(\pi g) \cos \left(u^{0}\right),
\end{aligned}
$$

where $g=3 A\left(128^{1 / 2} C\right)^{-1}, h^{*}=A^{3}\left(12 C^{2}\right)^{-1}$.

Thus for $h>h^{*}$, sufficiently small $u \neq 0$ and almost all ratios $A / C$ the Poincaré-Melnikov functions $M_{k}, k=1,2, \ldots, 13$, have simple zeros. Therefore the system (1) for the sets (23) contains transverse homoclinic orbits and hence a pathological structure.

\section{Concluding remarks}

The Poincaré-Melnikov method is a regular method, and establishes only the existence of nondegenerate homoclinic orbits, not their precise shape. The Poincare-Melnikov function technique shows that the maximal width of the splitting of the Lagrangian manifolds, and hence the width of the stochastic layer is $\mathrm{O}(\mu)$. However, there is little known about the precise relation between the splitting distance and angle and the thickness of the stochastic layers. It is known that the set of stochastic layers grows in size with $\mu$. On the other hand, there are examples that highly perturbed IIamiltonian systems become completely integrable (e.g. the deformations $(\mathrm{o}) \rightarrow$ (i), (o) $\rightarrow$ (ii), ..., (iii) $\rightarrow(\mathrm{o})$ ). The Lebesgue measure of the stochastic domain is still open question.

We have shown that the integrability of a family of Hamiltonian systems (1) is a discrete phenomenon in case (o) and (i), i.e. points $(A, B, C, D)$ for which the system is integrable form a zero measure set in $\mathrm{R}^{4}$.

In the limit $A \rightarrow \mu$ (case (o) in Sec. 2.1) we expect to see exponentially small separations of the manifolds $W^{s}\left(\boldsymbol{x}^{\mu}\right), W^{u}\left(\boldsymbol{x}^{\mu}\right)$, hence a failure of our regular perturbation theory. From Eqs. (9) and (17), we obtain that the splitting distance should be of the order

$$
d_{\max }=\mathrm{O}\left[\mu \exp \left(-\mu^{-1 / 2}\right)\right] \text {. }
$$


Since the splittings are exponentially small, perturbation expansions using series in $\mu$ will not succeed.

$$
\begin{aligned}
& \text { A little calculation shows that for the sets } \\
& (A, A, 15 A / 16+\mu, 5 A / 16),(A, A, 15 A / 16,5 A / 16+\mu), \\
& (A, A+\mu, 15 A / 32+\mu, 5 A / 32),(A+\mu, 15 A / 24+\mu, 5 A / 24+\mu), \\
& (A, A+\mu, 45 A / 23,15 A / 32+\mu),(A+\mu, A, 15 A / 24+\mu, 5 A / 24+\mu), \\
& (A, A+\mu, 15 A / 8+\mu, 5 A / 8),(A+\mu, A+\mu, 15 A / 16+\mu, 5 A / 16), \\
& (A+\mu, A+\mu, 15 A / 16,5 A / 16+\mu), \\
& (A+\mu, A+\mu, 15 A / 16+\mu, 5 A / 16+\mu),
\end{aligned}
$$

the Poincaré-Melnikov function vanishes identically, and the cxistence of transversal homoclinic intersections cannot be established.

Exponentially small splittings also occur in Kolmogorov-Arnold-Moser theory. While there is a general belief that this does reflect the correct picture (e.g. [14]), there is as yet no rigorous proof (cf. [15]).

There remains the open question whether the generalized IIenon-Heiles system has chaotic layers near other integrable cases (ii) and (iii). In case (ii) the second integral

$$
G=(4 A-B)\left(p_{1}^{2}+A p_{2}^{2}\right)+4 C\left(A q_{1}^{2}-p_{2}^{2}\right) q_{2}+C q_{2}^{2}\left(q_{1}^{2}+4 q_{2}^{2}\right)
$$

is quadratic in momenta which reflects separability of the Hamilton-Jacobi equation. For such class of potentials, as shown by Darboux, the IIamilton-Jacobi equation is separable in confocal elliptic coordinates (cf. [16]). Nevertheless, for this case the existence of chaotic behaviour cannot be proven analytically. An additional integral for case (iii):

$$
\begin{aligned}
G= & 3 p_{1}^{4}+6\left(A+2 C q_{2}\right) q_{1}^{2} p_{1}^{2}-4 C q_{1}^{3} p_{1} p_{2} \\
& +3 A^{2} q_{1}^{4}-4 C q_{1}^{4}\left(A q_{2}+C q_{2}^{2}\right)-C^{2} q_{1}^{6} / 6
\end{aligned}
$$

is a nonreducible quartic polynomial in momenta and therefore IIamiltonian (1) is not separable.

\section{References}

[1] M.C. Gutzwiller, Chaos in Classical and Quantum Mechanics, Springer-Verlag, Berlin 1989.

[2] J. Ford, Adv. Chem. Phys. 24, 155 (1973).

[3] P.L. Christiansen, J.C. Eilbeck, V.Z. Enolskii, Yu.B. Gaidai, Phys. Lett. A 166, 129 (1992).

[4] M. Henon, C. Heiles, Astron. J. 69, 73 (1964).

[5] Y. Aizawa, N. Saito, J. Phys. Soc. Jap. 32, 1636 (1972).

[6] B. Grammaticos, B. Dorizzi, R. Padjen, Phys. Lett. A 89, 111 (1982). 
[7] H. Poincaré, Les Méthodes Nouvelles de la Mécanique Celeste, Vol. 3, Gauthier-Villars, Paris 1899.

[8] Z. Nitecki, Differentiable Dynamics, M.I.T. Press, Cambridge 1971.

[9] P.J. Holmes, J.E. Marsden, Commun. Math. Phys. 82, 523 (1982).

[10] C. Robinson, Ergod. Th. Dynam. Syst. 8*, 395 (1988).

[11] S. Wiggins, Introduction to Applicd Nonlinear Dynamical Systems and Chaos, Springer-Verlag, Berlin 1990.

[12] P.J. Holmes, Physica D 5, 335 (1982).

[13] E.T. Whittaker, G.N. Watson, A Course of Modern Analysis, 4th ed., Cambridge Univ. Press, Cambridge 1935.

[14] B.V. Chirikov, Phys. Rep. 53, 263 (1979).

[15] P. Holmes, J. Marsden, J. Scheurle, Contemp. Math. 81, 213 (1988).

[16] E.T. Whittaker, A Treatise on the Analytical Dynamics of Particles and Rigid Bodies, 4th ed., Cambridge Univ. Press, Cambridge 1959. 